\section{Avaliação da Fidedignidade de um Instrumento Voltado à Satisfação do Doador de Sangue}

\section{Reliability of a Blood Donor Satisfaction Questionnaire}

\section{Vera Lucia Borges'}

Edson Zangiacomi Martinez ${ }^{2 *}$

Marise Helena Bendini' ${ }^{1}$

Maria Adelina Gaviolli Fortunato Costa ${ }^{1}$

\section{Sueli Cristina Leoni Ferreira'}

${ }^{1}$ Hemocentro - Centro Regional de Hemoterapia do Hospital das Clínicas da Faculdade de Medicina de Ribeirão Preto, Universidade de São Paulo, HCFRMP - USP

${ }^{2}$ Departamento de Medicina Social, Faculdade de Medicina de Ribeirão Preto, Universidade de São Paulo

\section{Resumo}

Objetivos: O objetivo do presente estudo é avaliar a fidedignidade de um instrumento destinado a mensurar a satisfação de doadores de sangue em um centro de hematologia e transfusão. Sujeitos e Métodos: Foi desenvolvido um questionário objetivando mensurar a satisfação dos doadores de sangue do Hemocentro/Ribeirão Preto, onde os mesmos classificaram 18 afirmativas em uma escala de Likert de 4 itens, variando de "totalmente errado" a "totalmente certo". Uma amostra de 407 doadores foi obtida sistematicamente, onde, a cada quatro pessoas que seqüencialmente compareceram para doação, o quarto era convidado a preencher o questionário. Uma amostra final de 407 doadores foi utilizada para a análise da fidedignidade do questionário e análises preliminares. A análise de correspondência (AC) foi utilizada para avaliação do grau de satisfação dos doadores para cada item. Resultados: Os participantes tinham em média 31,2 anos (desvio padrão de 9,13 e amplitude de 18 a 67 anos). Foi encontrado um coeficiente alfa de Cronbach de 0,97 , indicando uma consistência interna muito alta do questionário. A AC indicou que os itens com maior insatisfação são o acesso ao Hemocentro e o tempo gasto na doação. Itens de maior satisfação referem-se à confiança no serviço, funcionários e atendimento. A AC identificou que, de modo geral, as pessoas que estão doando sangue pela primeira vez tendem a uma menor satisfação. Conclusão: A alta consistência interna do questionário indica que os itens abordados têm alto poder de mensurar um mesmo objeto, mas ainda há a necessidade de estudar a validação do instrumento para avaliar se tal objeto é necessariamente a satisfação do doador.

Palavras-chave: Doadores de sangue. Fidedignidade de instrumento. Satisfação. 
Abstract

Objectives: The purpose of this study was to evaluate the reliability of a self-reporting instrument to measure the Portuguese-speaking blood donor satisfaction in a hematology and blood transfusion center. Subjects and Methods: Data were collected from 407 donors between February and May 2004 at Hemocentro - Ribeirão Preto, Brazil. The questionnaire included items concerning blood donor background characteristics. Eighteen items measuring donor satisfaction dealt with the quality of staff-donor relationship (range of responses from $1=$ negative to $4=$ positive). Item homogeneity was analyzed using Cronbach alpha coefficient. Data analysis was based on correspondence analysis. Results: The mean age of responding donors was 31.2 years (standard deviation 9.13 years, range $18-67$ years), about $65 \%$ were men and $35 \%$ were women. Cronbach alpha for the eighteen items measuring donor satisfaction was 0.97 . Results showed that patient satisfaction was highest in items pertaining to staff-donor relationship, and trust in the services and care provided. Blood donors reported the most dissatisfaction with the access to Hemocentro and the time spent in donation. Results also showed differences in satisfaction rates between first-time blood donors and people who already had donated blood. Conclusion: The internal consistency of the questionnaire indicates that its items have a high capacity to measure the same object. However, its validity still requires further studies to confirm if this object is in fact blood donor satisfaction.

Key Words: Blood donors. Reliability. Satisfaction.

\section{Introdução}

A participação da população na doação de sangue é fundamental para a manutenção dos estoques, buscando evitar que a demanda de solicitação de bolsas de sangue seja maior que a reposição do mesmo. A fidelização de doadores de sangue exige que os mesmos sintam-se seguros quanto ao processo de doação e satisfeitos durante o processo de atendimento. A certificação na Norma NBR ISO 9001:2000 ${ }^{1,2}$ pode ser vista como um compromisso das instituições de hemoterapia com os doadores, visando uma melhoria contínua na prestação de seus serviços.

Dentro desta busca continuada de melhoria, a Norma NBR ISO 9001:2000 exige que as necessidades dos clientes sejam determinadas e atendidas com o propósito de aumentar sua satisfação, bem como o desenvolvimento contínuo dos profissionais e a medição e análise de processos e seus resultados. Assim, para a certificação de uma unidade de hemoterapia pela Norma, tornam-se necessários levantamentos de informações que garantam o conhecimento e o monitoramento contínuo dos níveis de satisfação dos doadores em relação ao serviço prestado, de forma que sejam fornecidos dados para a tomada de ações que, por sua vez, possibilitem um número cada vez maior de doadores satisfeitos.

Um cliente é usualmente definido como o indivíduo que adquire um produto ou serviço de uma empresa ou instituição. Em um setor de saúde, este conceito de cliente é adaptável ao paciente, dado que ele busca na instituição a saúde como produto, ainda que este produto não seja um bem de consumo. $\mathrm{Na}$ literatura especializada, muitos estudos foram desenvolvidos objetivando descrever princípios metodológicos para o desenvolvimento de pesquisas e de instrumentos de avaliação de satisfação de pacientes quanto a serviços de saúde ${ }^{3-9}$. Entretanto, a adaptação do conceito usual de cliente ao doador de sangue exige algum cuidado. $\mathrm{O}$ doador de sangue não é perfeitamente comparável a um consumidor, já que não está necessariamente adquirindo um bem, mas doando algo - 0 
seu sangue. Isto faz com que a avaliação da satisfação do doador se volte totalmente ao seu contentamento em relação ao serviço prestado, e não em relação a um possível produto adquirido. Esta certa incompatibilidade entre conceitos de doador de sangue e paciente faz com que muitos dos instrumentos já desenvolvidos para a mensuração da satisfação de um usuário de um serviço de saúde não sejam diretamente aplicáveis a doadores de sangue.

Com a motivação de atender ao requisito da Norma NBR ISO 9001:2000 que trata do monitoramento contínuo da satisfação do cliente, o presente trabalho objetiva a análise da fidedignidade ${ }^{10}$ de um instrumento voltado especificamente à mensuração da satisfação do doador de sangue quanto ao serviço prestado por uma unidade de hemoterapia.

Em um estudo sobre a implantação do Sistema de Gestão da Qualidade ISO no Hemocentro da Universidade Estadual de Campinas, Vicente ${ }^{11}$ (2002) utilizou uma ficha de avaliação de atendimento aos doadores (FAAD). A FAAD permite ao doador avaliar o atendimento em setores específicos de um serviço de hemoterapia (apoio ao doador, recepção, pré-triagem, triagem, coleta, copa), horário de funcionamento, área física e tempo de permanência. Os doadores de sangue avaliam tais aspectos em uma escala de quatro itens, de "ruim" a "ótimo". Neste estudo, mais de 24 mil doadores preencheram a FAAD, sendo o horário de atendimento e o tempo de permanência os aspectos com maiores índices de insatisfação.

Enquanto a FAAD mede a percepção do doador principalmente quanto à qualidade do atendimento, o presente trabalho busca explorar a satisfação neste e em outros domínios, como a acessibilidade e a confiança na equipe profissional.

\section{Sujeitos e Métodos}

\section{O questionário de satisfação do doador}

Donabedian $^{12}$ (1988), Fitzpatrick ${ }^{4}$ (1991) e Goldstein et $\mathrm{al}^{13}$ (2000) mencionam que a satisfação do paciente pode pertencer a distintos domínios. No desenvolvimento do nosso questionário, investigamos a satisfação segundo três domínios: acessibilidade/ conveniência, aspectos técnicos e aspectos interpessoais. No domínio acesso/conveniência, quatro itens são abordados: a facilidade para chegar ao Hemocentro, o tempo gasto para a doação, a rapidez no atendimento e a facilidade para encontrar vagas no estacionamento. A demora no atendimento é motivo comum de reclamações em serviços de hemoterapia, como indicado por Vicente $^{11}$ (2002). Eilers ${ }^{14}$ (2004) também apontou o tempo de espera como um significativo componente da satisfação do paciente. No domínio que envolve aspectos técnicos, são abordados itens em relação à limpeza do Hemocentro, a confiabilidade no material utilizado, o lanche oferecido e o espaço físico. O terceiro domínio trata do contentamento do doador com o contato com os funcionários do Hemocentro. Alguns itens abordam diretamente $o$ atendimento dos funcionários segundo seu setor de trabalho: a recepção, a assistência social e a coleta de sangue. Outros itens abordam a percepção do doador quanto ao prazer dos funcionários em atendê-lo e explicar suas dúvidas, à gentileza, ao sentir-se à vontade $\mathrm{e}$ ao respeito.

Os itens que integram estes três domínios foram transcritos sob a forma de 18 afirmativas, conforme mostrado no Quadro 1. Um sorteio determinou a ordem dos itens no questionário. Os doadores de sangue eram convidados a classificar estas afirmativas em uma escala de Likert ${ }^{15}$ de 4 itens, variando de "totalmente errado" a "totalmente certo". Dado que as afirmativas foram escritas sempre de maneira positiva, nota-se que a resposta "totalmente errado" reflete uma insatisfação em relação ao respectivo item, enquanto a resposta "totalmente certo” reflete satisfação em relação ao mesmo. Também foi incluído no questionário um cabeçalho, onde o doador informava o seu sexo, a idade, o grau de escolaridade (segundo as categorias "sem escolaridade", " $1^{\circ}$ grau”, " $2^{\circ}$ grau” e "superior”) e se compare- 
cia para sua primeira doação ou não. Outras duas questões questionavam o motivo pelo qual o respondente escolheu o Hemocentro para a doação de sangue e quais os sentimentos experimentados durante a doação. Não foi incluído nenhum campo onde o doador pudesse se identificar.

O questionário foi construído com a pretensão de ser auto-explicativo, ou seja, o doador poderia preenchê-lo sem o auxílio de um funcionário do Hemocentro. Até a obtenção da versão final do questionário, algumas versões iniciais foram testadas, sofrendo revisões sucessivas de acordo com as limitações observadas.

\section{Amostra e coleta de dados}

Os dados foram coletados no Hemocentro de Ribeirão Preto (Centro Regional de Hemoterapia do Hospital das Clí- nicas da Faculdade de Medicina de Ribeirão Preto, Universidade de São Paulo, HCFRMP - USP), entre os meses de fevereiro a maio de 2004. A amostragem foi feita de forma sistemática, onde, a cada quatro doadores que seqüencialmente compareciam ao Hemocentro, um era convidado a preencher o questionário. Foram incluídos na amostra somente doadores alfabetizados. Os questionários foram entregues aos doadores que concordaram em participar do estudo na lanchonete do Hemocentro, que constitui a última etapa do fluxo da doação. Após o preenchimento do questionário, os participantes o depositavam em uma urna. A coleta de dados foi realizada de segunda-feira a domingo, em duas semanas alternadas a cada mês, durante todo o período de atendimento (das 7 às 13 horas). Os questionários eram recolhidos diariamente e digitados no programa EpiInfo 6.

Quadro 1 - Itens abordados no questionário de satisfação de doadores, seus rótulos utilizados na análise de correspondência e a dimensão da satisfação a qual pertencem.

Chart 1 - Items included in the blood-donor satisfaction questionnaire, the labels used in the correspondence analysis, and the dimension of satisfaction they belong to.

\begin{tabular}{|c|c|c|}
\hline Rótulo & Item & o da satisfação \\
\hline A & É fácil chegar ao Hemocentro & ACES \\
\hline B & Eu gastei pouco tempo para doar & ACES \\
\hline C & O Hemocentro é um lugar limpo & $\mathrm{T}$ \\
\hline D & Aqui as pessoas parecem que gostam de me atender & $\mathrm{IP}$ \\
\hline $\mathrm{E}$ & Eu acredito na qualidade do material utilizado aqui & $\mathrm{T}$ \\
\hline $\mathrm{F}$ & Eu gostei do lanche que me ofereceram & $\mathrm{T}$ \\
\hline G & Eu fui rapidamente atendido durante a doação de sangue & ACES \\
\hline $\mathrm{H}$ & O Hemocentro é um lugar espaçoso & $\mathrm{T}$ \\
\hline I & Aqui as pessoas são gentis comigo & IP \\
\hline J & Eu me senti à vontade durante a entrevista & $\mathbb{I P}$ \\
\hline $\mathrm{K}$ & Aqui as pessoas explicam tudo aquilo que quero entender & $\mathrm{IP}$ \\
\hline $\mathrm{L}$ & Eu confio na capacidade profissional dos funcionários do Hemocentro & $\mathbb{I P}$ \\
\hline M & Aqui sinto que as pessoas me tratam com respeito & $\mathrm{IP}$ \\
\hline $\mathrm{N}$ & Eu fui bem atendido pelo funcionário que fez os exames antes da entrevista & $\mathbb{I P}$ \\
\hline $\mathrm{O}$ & Eu fiquei satisfeito com o atendimento do funcionário que me atendeu na recepção & $\mathbb{I P}$ \\
\hline$P$ & Eu gostei do atendimento da Assistente Social e suas orientações & $\mathbb{I P}$ \\
\hline Q & Foi fácil encontrar vagas no estacionamento & ACES \\
\hline $\mathrm{R}$ & Eu gostei do atendimento do funcionário que colheu meu sangue & IP \\
\hline
\end{tabular}

ACES: acessibilidade/conveniência; T: técnico; IP: interpessoal 


\section{Análise dos dados}

$\mathrm{Na}$ análise da consistência interna do questionário foi utilizado o coeficiente alfa de Cronbach ${ }^{16,17}$, e na análise preliminar dos dados utilizou-se a análise de correspondências simples ${ }^{18-20}$.

A análise de correspondências (AC), chamada por alguns autores de análise fatorial de correspondências, é uma técnica estatística multivariada que permite medir e visualizar, de modo gráfico, o grau de associação entre um conjunto de categorias de variáveis qualitativas. Quando aplicada aos dados deste questionário de avaliação da satisfação do doador, indica quais são os itens associados a cada diferente categoria de satisfação em um gráfico cartesiano denominado mapa de correspondências. Na AC, a variação total dos dados é denominada inércia, sendo esta variação decomposta em cada eixo (ou dimensão) do gráfico. Assim, a inércia associada a cada dimensão do gráfico nos informa qual é a proporção da variação total que aquele eixo está explicando.

Em uma análise inicial, observou-se que a resposta "totalmente errado" apresentava uma alta proximidade com a resposta "errado" no mapa de correspondências. Assim, as respostas "totalmente errado" e "errado" foram unidas em uma única categoria. Em relação a cada item, o doador foi então classificado em "insatisfeito" se respondeu "totalmente errado" ou "errado", em "satisfeito" se respondeu "certo", ou "muito satisfeito" se respondeu "totalmente certo". Quando um doador não respondeu a algum item, atribuiu-se uma quarta classificação, incluída na AC como um ponto suplementar ${ }^{18}$. Um ponto suplementar é um elemento que não intervém na análise, não participa da formação dos eixos, mas pode ser representado no mapa de correspondência de modo que sua localização seja um auxílio à interpretação.

Uma AC para dados com estrutura de grupos, conforme proposta por Pamplona ${ }^{21}$, avaliou se a satisfação é influenciada pelo fato de o indivíduo estar ou não doando san- gue pela primeira vez. Esta técnica permite a inserção de uma variável de controle na AC, e tem a vantagem de manter a inércia encontrada na AC simples.

As freqüências de sentimentos relatados e motivações ${ }^{22}$ para a doação de sangue foram comparadas entre grupos de indivíduos que doavam pela primeira vez e indivíduos que já haviam doado anteriormente pelo teste exato de Fisher, adotando um nível de significância de 0,05. Todas as análises foram desenvolvidas com o programa de computador SAS versão 8.

\section{Resultados}

A análise da fidedignidade do questionário e a análise preliminar dos dados utilizaram uma amostra final de 407 doadores. Esta amostra foi composta por 189 (65\%) homens e 101 mulheres, sendo que 117 doadores ( $29 \%$ do total) não informaram o sexo. Estavam doando sangue pela primeira vez 120 pessoas, 228 já haviam doado sangue e 59 ( $15 \%$ do total) não forneceram tal informação. Catorze respondentes não têm escolaridade, 130 cursaram o primeiro grau, $146 \mathrm{o}$ segundo grau, 57 têm curso superior e 60 ( $14,7 \%$ do total) não forneceram informações sobre seu nível de escolaridade. A Tabela 1 mostra os sentimentos que os participantes referiram experimentar quando doaram sangue e suas motivações da escolha do Hemocentro para a doação, segundo a informação de estarem ou não doando sangue pela primeira vez. Cada doador poderia relatar mais de um sentimento e/ou mais de uma motivação. Nota-se que os sentimentos de ansiedade e medo estão significativamente mais presentes entre novos doadores. Dentre os indivíduos que relataram estar doando sangue a pedido de parente ou amigo, há uma freqüência significativamente maior de novos doadores $(p=0,02$, sendo $61,7 \%$ de novos doadores contra $48,7 \%$ de indivíduos que já haviam doado sangue).

A consistência interna do questionário, quanto aos 18 itens que pretendem avaliar a satisfação do doador (listados na Tabela 2, onde a cada item foi atribuído um rótulo de 
Tabela 1 - Sentimentos que os participantes referiram experimentar quando doaram sangue e suas motivações da escolha do Hemocentro para a doação, segundo a informação de estarem ou não doando pela primeira vez.

Table 1 - Feelings donors reported when they donated blood and the reasons they chose Hemocentro for donation, according to whether they were donating for the first time or not.

\begin{tabular}{|c|c|c|c|}
\hline & \multicolumn{2}{|c|}{ Primeira doação? } & \multirow{2}{*}{$\begin{array}{l}\text { Teste exato de } \\
\text { Fisher ( } p \text { valor })\end{array}$} \\
\hline & $\operatorname{sim}(\%)$ & não(\%) & \\
\hline \multicolumn{4}{|c|}{ Sentimentos experimentados quando doou sangue } \\
\hline Sensação de dever cumprido & 50,0 & 60,1 & 0,09 \\
\hline Ansiedade & 16,7 & 6,1 & $<0,01$ \\
\hline Alegria & 44,2 & 51,3 & 0,22 \\
\hline Medo & 5,8 & 1,3 & 0,04 \\
\hline Prazer & 34,2 & 43,0 & 0,13 \\
\hline Dor & 2,5 & 1,8 & 0,70 \\
\hline Irritação & 1,7 & 0,4 & 0,27 \\
\hline Sentiu-se importante & 39,2 & 50,4 & 0,05 \\
\hline \multicolumn{4}{|c|}{ Motivações da escolha do Hemocentro para a doação } \\
\hline Foi solicitado pelo Hemocentro & 14,5 & 13,2 & 0,74 \\
\hline Pedido de parente ou amigo & 61,7 & 48,7 & 0,02 \\
\hline Sente-se seguro & 45,8 & 69,7 & $<0,01$ \\
\hline É perto de onde mora ou trabalha & 21,0 & 20,2 & 0,89 \\
\hline É bem tratado aqui & 45,8 & 61,4 & $<0,01$ \\
\hline Não conhece outro serviço & 10,0 & 7,0 & 0,41 \\
\hline
\end{tabular}

'A' a 'R'), foi medida pelo coeficiente alfa de Cronbach $^{16,17}$. Um coeficiente alfa de 0,965 indicou uma consistência interna muito alta, ou seja, os 18 itens formam um conjunto consistente no sentido de mensurar um mesmo objeto, que almeja ser a satisfação do doador.

Outras informações sobre o comportamento de cada item utilizado no cálculo do coeficiente alfa de Cronbach estão listadas na Tabela 2. A correlação entre o item e o total é o coeficiente de correlação entre o item específico e o indicador total, depurado de sua própria contribuiçãao ${ }^{23}$. Esta correlação mede a associação do item com as outras medidas tomadas em conjunto. Notase que os itens A e B, ou seja, a facilidade para chegar ao Hemocentro e o tempo gasto na doação, respectivamente, possuem menor correlação entre o item e o total. Isto não significa que estes itens não sejam úteis na avaliação da satisfação, mas é uma evidência de que estejam avaliando a satisfação sob um aspecto diferente dos demais. $\mathrm{O}$ alfa de Cronbach calculado quando o respectivo item é desprezado mostra o impacto que teria a retirada deste item do questionário ${ }^{23}$. Na Tabela 2, nota-se que a eliminação de qualquer um dos itens não implica em uma significativa mudança do alfa de Cronbach, previamente calculado em 0,965 . Isso é uma evidência de que nenhum item do questionário estaria mensurando algum aspecto não relacionado ao objeto que pretende ser a satisfação do doador.

A Figura 1 mostra o mapa de correspondências gerado da análise dos dados, onde se verifica que as respostas "muito satisfeito", "satisfeito" e "insatisfeito" são representadas nesta ordem quando projetadas na dimensão 1. Esta dimensão é responsável por 90,2\% da inércia, ou seja, $90,2 \%$ da variação total dos dados são explicados por este eixo do gráfico. Cada item do questionário é representado no mapa de correspondência por seu rótulo (de 'A' a 'R', conforme a Tabela 2), sendo que a correspondência de um item com as respostas "muito satisfeito", "satisfeito" ou "insatisfeito" é representada por sua distância no gráfico. Assim, os itens associa- 
Tabela 2 - Itens constantes do questionário de satisfação de doadores e resultados do seu exame de consistência interna

Table 2 - Items included in the blood-donor satisfaction questionnaire and results of the internal consistency test.

\begin{tabular}{llcc}
\hline & Item & $\begin{array}{c}\text { Correlação entre } \\
\text { o item e o total }\end{array}$ & $\begin{array}{c}\text { Alfa de Cronbach } \\
\text { se o item é desprezado }\end{array}$ \\
\hline A & facilidade para chegar ao Hemocentro & 0,5390 & 0,9667 \\
B & tempo gasto na doação de sangue & 0,5991 & 0,9659 \\
C & limpeza do Hemocentro & 0,7487 & 0,9637 \\
D & o prazer dos funcionários em atender & 0,7626 & 0,9635 \\
E & confiança na qualidade do material utilizado & 0,7998 & 0,9630 \\
F & lanche oferecido & 0,6382 & 0,9652 \\
G & rapidez no atendimento durante a doação de sangue & 0,7398 & 0,9638 \\
H & espaço físico do Hemocentro & 0,7242 & 0,9641 \\
I & gentileza dos funcionários & 0,8402 & 0,9625 \\
J & o sentir-se à vontade durante a entrevista & 0,8595 & 0,9622 \\
K & explicações dos funcionários & 0,8618 & 0,9622 \\
L & capacidade profissional dos funcionários & 0,8582 & 0,9622 \\
M & o respeito no tratamento & 0,8468 & 0,9624 \\
N & atendimento do funcionário que faz os exames & 0,8785 & 0,9620 \\
O & satisfação com o funcionário da recepção & 0,8192 & 0,9627 \\
P & atendimento e orientações da assistente social & 0,8499 & 0,9623 \\
Q & facilidade de encontrar vagas no estacionamento & 0,7089 & 0,9645 \\
R & atendimento do funcionário que colhe o sangue & 0,7807 & 0,9633 \\
\hline
\end{tabular}

dos a um maior grau de insatisfação têm seus rótulos representados à direita do gráfico, próximos ao rótulo "insatisfeito". Por sua vez, os itens associados a um maior grau de satisfação aparecem à esquerda do gráfico, próximos ao rótulo "muito satisfeito". Notamos que os itens com maior grau de insatisfação são os rotulados por A e B (a facilidade para chegar no Hemocentro e o tempo gasto na doação de sangue, respectivamente),

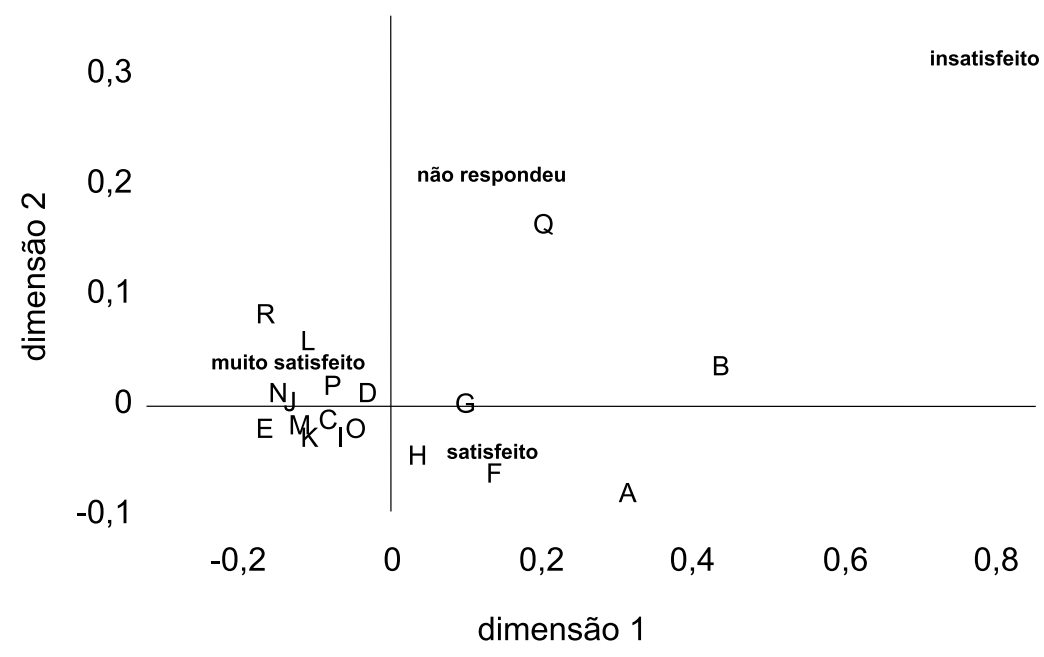

Figura 1 - Visualização das associações entre os itens abordados no questionário de satisfação de doadores de sangue e o grau de satisfação, por intermédio do mapa gerado pela análise de correspondências simples.

Figure 1-Image of the items included in the blood-donor satisfaction questionnaire and the level of satisfaction in a map plotted through simple correspondence analysis. 
aqueles com média satisfação são os rotulados por F, G, H e Q, e os avaliados com maior grau de satisfação são os rotulados por $C$, D, E, I, J, K, L, M, N, O, P e R. É interessante notar que, relativamente, os itens pertencentes ao domínio acessibilidade/conveniência (A, B, G e Q) são classificados como aspectos de maior insatisfação quando comparados aos itens dos domínios de aspectos técnicos (C, E, F e H) e interpessoais (os demais itens, conforme Quadro 1).

A dimensão 2 é responsável por apenas $9,8 \%$ da inércia. Neste eixo destaca-se apenas o item $\mathrm{Q}$, referente à facilidade de encontrar vagas no estacionamento, localizado com maior proximidade da representação "não respondeu". Este efeito é natural, dado que uma grande parte dos doadores não utiliza o estacionamento do Hemocentro e, portanto, não responde a este item. Verificamos que a projeção das coordenadas dos demais itens sobre a dimensão 2 do gráfico não tem uma variação importante (encontram-se todos representados em uma faixa estreita do eixo, de $-0,1$ a 0,1), indicando que a freqüência de não respostas é homogênea entre estes.

Uma segunda análise de correspondências objetivou avaliar se o fato do indivíduo estar se apresentando para a sua primeira doação ou já ter doado sangue alguma vez exerce alguma influência na satisfação. $\mathrm{O}$ mapa de correspondências gerado por esta análise com estrutura de grupos ${ }^{21}$ é representado na Figura 2, onde os rótulos representados por letras maiúsculas refletem os itens respondidos pelos indivíduos que doavam sangue pela primeira vez, e as letras minúsculas associam-se aos itens respondidos pelos indivíduos com alguma doação anterior. Esta AC foi baseada em uma amostra de 348 doadores de sangue, dado que 59 doadores não informaram se estavam ou não doando sangue pela primeira vez. A primeira dimensão é responsável por $73,7 \%$ da inércia, e a segunda dimensão, por 26,3\%. Neste mapa, observa-se que os indivíduos que já haviam doado sangue mostram maior grau de satisfação que os novos doadores em relação a todos os itens do questionário, com

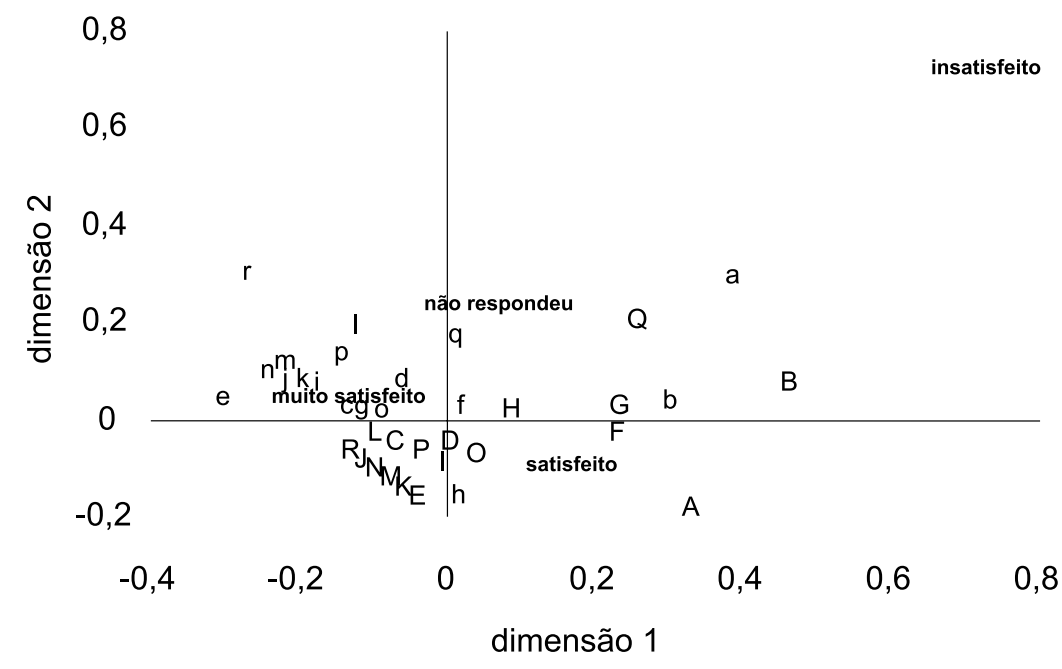

Figura 2 - Visualização das associações entre os itens abordados no questionário de satisfação de doadores de sangue e o grau de satisfação, por intermédio do mapa gerado pela análise de correspondências com estrutura de grupos. Os itens respondidos por indivíduos que doavam sangue pela primeira vez são representados por letras maiúsculas, enquanto os itens respondidos por indivíduos que já haviam doado sangue anteriormente são representados por letras minúsculas.

Figure 2 - Image of the associations between the items included in the blood-donor satisfaction questionnaire and the level of satisfaction, in a map plotted through correspondence analysis with group structure. The items answered by first-time donors are represented in upper case, while the items answered by repeat donors are represented in lower case. 
exceção do item A (facilidade para chegar no Hemocentro). Isto é verificado no mapa pelas posições dos rótulos representados pelas letras minúsculas, que aparecem no gráfico sempre à esquerda de seus respectivos pares maiúsculos. Da aplicação de testes estatísticos de hipóteses, verificamos que o grau de satisfação é significativamente diferente (respectivos $p$ valores menores que 0,05, de acordo com testes exatos de Fisher) entre novos doadores e indivíduos que já doaram sangue segundo os itens A (facilidade para chegar no Hemocentro), E (confiança na qualidade do material utilizado na doação), G (rapidez na doação de sangue), $\mathrm{M}$ (respeito no tratamento), $\mathrm{N}$ (atendimento do funcionário que fez os exames) e $\mathrm{R}$ (atendimento do funcionário que colhe o sangue).

\section{Conclusões}

Ainda que o questionário apresente alta consistência interna, o que pode ser interpretado como um indicativo de que os itens abordados têm alto poder de mensurar o mesmo objeto, ainda existe a necessidade de estudar a validação do instrumento. Trabalhos futuros deverão avaliar se este objeto reflete necessariamente a satisfação do doador de sangue, através do exame da sua concordância com uma possível medida de referência para satisfação. Dentro de um processo de melhoria contínua, é importante uma busca constante de aspectos a serem aperfeiçoados. Observando que um importante objetivo do instrumento dentro do monitoramento contínuo da qualidade não é medir com generalidade o nível de satisfação do doador, mas conhecer os itens específicos que merecem atenção na busca da melhoria contínua da qualidade, novas mudanças poderiam incluir novos itens, retirando-se outros que ao longo do tempo forem sanados e deixarem de ser apontados como fatores de maior insatisfação. Para alcançar este objetivo, a validação do instrumento deve também prever a análise de itens específicos.

Os resultados deste estudo evidenciam que os indivíduos que comparecem à instituição para doar sangue pela primeira vez e aqueles que já doaram em alguma ocasião constituem dois perfis distintos de doadores. Desta forma, uma pesquisa de satisfação de doadores de sangue deve sempre levar em consideração a possibilidade de resultados distintos entre estes diferentes perfis.

\section{Referências}

1. ABNT - Associação Brasileira de Normas Técnicas NBR ISO 9002 - Modelo para a garantia da qualidade em produção, instalação e serviços associados. Rio de Janeiro: ABNT; 1994.

2. ABNT - Associação Brasileira de Normas Técnicas NBR ISO 9001:2000 - Sistemas de Gestão da Qualidade Requisitos. Rio de Janeiro: ABNT; 2001.

3. La Monica EL, Oberst MT, Madea AR, Wolf RM. Development of a patient satisfaction scale. Res Nurs Health 1986, 9: 43-50.

4. Fitzpatrick R. Surveys of patient satisfaction: I Important general considerations. Br Med J 1991, 302: 887-9.

5. Fitzpatrick R. Surveys of patient satisfaction: II Designing a questionnaire and conducting a survey. $\mathrm{Br}$ Med J 1991; 302: 1129-32.

6. Sitzia J, Wood N. Patient satisfaction: a review of issues and concepts. Soc Sci Med 1997; 45: 1829-43.
7. Harris LE, Swindle RW, Mungai SM, Weinberger M, Tierney WM. Measuring patient satisfaction for quality improvement. Med Care 1999; 37: 1207-13.

8. Andaleeb SS. Service quality perceptions and patient satisfaction: a study of hospitals in a developing country. Soc Sci \& Med 2001; 52: 1359-70.

9. Bone MR. Surveys of patient satisfaction. Br Med J 1991; 303: 60.

10. Anastasi A, Urbina S. Fidedignidade. In: Testagem Psicológica. Porto Alegre: Artmed; 2000. p. .84-105.

11. Vicente CS. Implantação de um sistema de Gestão da Qualidade Internacional ISO no Hemocentro da UNICAMP e seu impacto na sistematização e melhoria da assistência de enfermagem aos doadores de sangue [dissertação de mestrado]. Campinas: Faculdade de Engenharia Mecânica da Universidade Estadual de Campinas; 2002.

12. Donabedian A. The quality of care: how can it be assessed? J Am Med Assoc 1988; 260: 1743-8. 
13. Goldstein MS, Elliott SD, Guccione AA. The development of an instrument to measure satisfaction with physical therapy. Phys Ther 2000; 80: 853-63.

14. Eilers GM. Improving patient satisfaction with waiting time. J Am Coll Health 2004; 53: 41-3.

15. Likert R. A technique for the measurement of attitudes. Arch Psychol 1932; 140: 55.

16. Cronbach LJ. Coefficient alpha and the internal structure of tests. Psychometrika 1951; 16: 297-334.

17. Bland JM, Altman DG. Cronbach's alpha. Br Med J 1997; 314: 572 .

18. Greenacre MJ. Correspondence analysis in practice. London: Academic Press; 1993.

19. Greenacre MJ. Theory and applications of correspondence analysis. London: Academic Press; 1984.
20. Greenacre MJ, Hastie T. The geometric interpretation of correspondence analysis. J Am Stat Assoc 1987; 82: 437-44.

21. Pamplona AS. Análise de correspondência para dados com estrutura de grupo [dissertação de mestrado]. Campinas: Instituto de Matemática, Estatística e Computação Científica da Universidade Estadual de Campinas; 1998.

22. Glynn AS, Kleinman SH, Schreiber GB, Zuck T, McCombs S, Bethel J, Garratty G, Williams AE. Motivations to donate blood: demographic comparisons. Transfusion 2002; 42: 216-25.

23. Pereira JCR. Análise de dados qualitativos: estratégias metodológicas para as Ciências da Saúde, Humanas e Sociais. 3.ed. São Paulo: Editora da Universidade de São Paulo; 2001.

recebido em: 20/11/04 versão final reapresentada em: 18/05/05 aprovado em: 06/06/05 